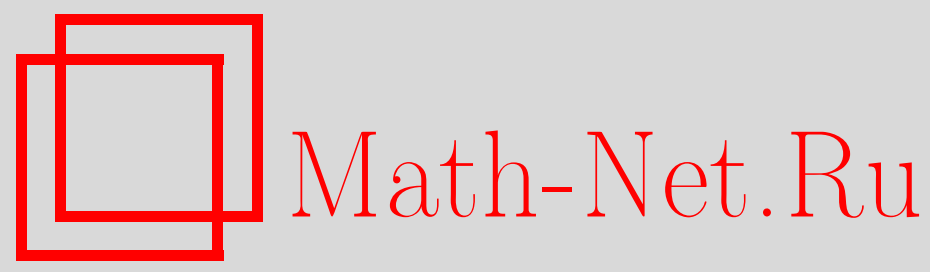

C. С. Марченков, Задание позитивно замкнутых классов посредством полугрупп эндоморфизмов, Дискрет. матем., 2012, том 24, выпуск 4, 19-26

DOI: https://doi.org/10.4213/dm1207

Использование Общероссийского математического портала Math-Net.Ru подразумевает, что вы прочитали и согласны с пользовательским соглашением http://www . mathnet.ru/rus/agreement

Параметры загрузки:

IP : 54.209 .52 .79

26 апреля 2023 г., 14:31:03 


\title{
Задание позитивно замкнутых классов посредством полугрупп эндоморфизмов
}

\author{
() 2012 г. . С. Марченков
}

\begin{abstract}
Позитивно замкнутые классы многозначной логики характеризуются с помощью полугрупп эндоморфизмов. Описываются все подполугруппы симметрической полугруппы степени $k$, которые являются полугруппами эндоморфизмов позитивно замкнутых классов в $P_{k}$. На основе полученных результатов находятся все позитивно предполные классы в $P_{k}$.

Работа выполнена при поддержке Российского фонда фундаментальных исследований, проект 10-01-00768.
\end{abstract}

Среди способов классификации множества функций многозначной логики важное место занимают способы, базирующиеся на операторах замыкания. На множестве $P_{k}$ функций $k$-значной логики задается оператор $\mathcal{O}$, и тогда множества функций из $P_{k}$, замкнутые относительно оператора $\mathcal{O}$ (обычно их называют $\mathcal{O}$-замкнутыми классами), образуют $\mathcal{O}$ классификацию множества $P_{k}$.

Наиболее известной классификацией этого типа является классификация, основанная на операторе (операции) суперпозиции. Эта классификация довольно давно и интенсивно изучается. Однако на пути полного описания классов в данной классификации имеется объективное препятствие: при любом $k \geqslant 3$ число замкнутых классов в $P_{k}$ континуально [10]. В связи с этим разными авторами и на основе различных идей предлагались “сильные” операторы замыкания, которые при любом $k$ порождают на множестве $P_{k}$ конечные либо счетные классификации.

Одним из первых сильных операторов замыкания стал оператор параметрического замыкания, введенный А. В. Кузнецовым [3]. Все 25 параметрически замкнутых классов булевых функций найдены в [3] (см. также [5]); конечность числа параметрически замкнутых классов в $P_{3}$ доказана в [2], а для $P_{k}$ при $k \geqslant 4-$ в [11]. Стоит отметить, что при $k \geqslant 3$ полного описания параметрически замкнутых классов в $P_{k}$ пока не получено.

Несколько позднее в [4] (см. также [5]) было предложено более формализованное логико-функциональное определение оператора параметрического замыкания. Это определение позволило ввести еще несколько новых сильных операторов замыкания. Первым среди них стал оператор позитивного замыкания (который оказался еще более “сильным”, нежели оператор параметрического замыкания). Оператор позитивного замыкания исследовался автором в [4-9]. В частности, было найдено (см. [4]), что имеется ровно шесть позитивно замкнутых классов булевых функций и при любом $k \geqslant 3$ число позитивно замкнутых классов в $P_{k}$ строго меньше числа подполугрупп с единицей в симметрической полугруппе степени $k$. 
В настоящей работе мы хотим охарактеризовать позитивно замкнутые классы в духе теории Галуа для алгебр Поста [1]. Как оказалось, подходящим средством для описания позитивно замкнутых классов являются двуместные отношения специального вида - графики одноместных функций. Более того, множество всех одноместных функций, которое соответствует данному позитивно замкнутому классу $Q$, образует полугруппу с единицей - полугруппу эндоморфизмов класса $Q$.

В теореме 1 найдена связь между полугруппой эндоморфизмов произвольного множества функций и позитивно замкнутым классом, который позитивно порождается этим множеством функций. Теорема 2 описывает в точности те полугруппы, которые являются полугруппами эндоморфизмов позитивно замкнутых классов. В теореме 3 на основе теоремы 1 находятся все позитивно предполные классы в $P_{k}$.

Введем необходимые понятия. Пусть $k \geqslant 2, E_{k}=\{0,1, \ldots, k-1\}, P_{k}-$ множество всех функций на $E_{k}$ (множество функций $k$-значной логики). Если $Q \subseteq P_{k}$, то через $Q^{(n)}$ обозначаем множество всех $n$-местных функций из $Q$. Если $Q \subseteq P_{k}, g \in P_{k}^{(1)}$ и для всякой функции $f$ из $Q$ выполняется тождество

$$
f\left(g\left(x_{1}\right), \ldots, g\left(x_{n}\right)\right)=g\left(f\left(x_{1}, \ldots, x_{n}\right)\right),
$$

то говорят, что $g$ есть эндоморфизм алгебры $\left\langle E_{k} ; Q\right\rangle$. С чуть меньшей строгостью будем также говорить, что $g$ есть эндоморфизм множества функций $Q$. Множество $\operatorname{End}\left\langle E_{k} ; Q\right\rangle$ всех эндоморфизмов алгебры $\left\langle E_{k} ; Q\right\rangle$ образует моноид - полугруппу с операцией композиции (суперпозиции) и единицей (тождественной функцией). В дальнейшем множество всех эндоморфизмов алгебры $\left\langle E_{k} ; Q\right\rangle$ будем обозначать через $\operatorname{End}(Q)$, а в случае $Q=\{f\}$ - через $\operatorname{End}(f)$.

Напомним основные понятия, связанные с оператором позитивного замыкания [4]. Вначале определим язык Pos. Исходными символами языка Рos являются символы предметных переменных $x_{0}, x_{1}, \ldots$ (с областью значений $E_{k}$ ), символы $f_{i}^{(n)}$ для обозначения $n$-местных функций из $P_{k}, 1 \leqslant i \leqslant k^{k^{n}}, n=1,2 \ldots$, знаки равенства $=$, конъюнкции $\&$, дизъюнкции $\vee$, квантора существования $\exists$, левой и правой скобок и запятой. Иногда вместо символов переменных $x_{0}, x_{1}, \ldots$ будем использовать символы $x, y, z$, а вместо символов $f_{i}^{(n)}$ - символы $g, h$.

Терм языка Pos определим по индукции. Символ предметной переменной есть терм; если $x_{j_{1}}, \ldots, x_{j_{n}}-$ символы предметных переменных (не обязательно различные), а $f_{i}^{(n)}$ - символ $n$-местной функции, то $f_{i}^{(n)}\left(x_{j_{1}}, \ldots, x_{j_{n}}\right)$ есть терм; если $t_{1}, \ldots, t_{m}$ - термы, а $f_{l}^{(m)}-$ символ $m$-местной функции, то $f_{l}^{(m)}\left(t_{1}, \ldots, t_{m}\right)$ есть терм. Других термов в языке Ров нет.

Всякий терм языка Pos очевидным образом определяет некоторую функцию класса $P_{k}$ (переменная определяет тождественную функцию).

Если $t_{1}, t_{2}$ - термы языка Pos, то выражение $\left(t_{1}=t_{2}\right)$ называем элементарной формулой языка Pos. Далее, если $\Phi_{1}, \Phi_{2}-$ формулы языка Pos, a $x_{i}-$ символ предметной переменной, то $\left(\Phi_{1} \& \Phi_{2}\right),\left(\Phi_{1} \vee \Phi_{2}\right),\left(\exists x_{i}\right) \Phi_{1}$ - также формулы языка Pos. Понятия свободной и связанной переменных предполагаем известными.

Всякая формула языка Pos c $m$ свободными переменными определяет некоторое $m$-местное отношение на $E_{k}$. Пусть $Q \subseteq P_{k}, \Phi\left(x_{1}, \ldots, x_{m}\right)$ - формула языка Pos co свободными переменными $x_{1}, \ldots, x_{m}$, все функциональные символы которой суть обозначения функций из $Q$, и формула $\Phi\left(x_{1}, \ldots, x_{m}\right)$ определяет отношение $r\left(x_{1}, \ldots, x_{m}\right)$ на $E_{k}$. В этом случае говорим, что формула $\Phi$ позитивно выражает отношение $r$ через функции множества $Q$. Понятие позитивной выразимости перенесем с отношений 
на функции. Именно, если $g\left(x_{1}, \ldots, x_{m}\right)-$ функция из $P_{k}$, а формула $\Phi\left(x_{1}, \ldots, x_{m}, y\right)$ языка Pos позитивно выражает отношение $y=g\left(x_{1}, \ldots, x_{m}\right)$ (график функции $g$ ) через функции множества $Q$, то говорим, что формула $\Phi$ позитивно выражает функцию $g$ через функции множества $Q$. Совокупность всех функций, позитивно выразимых через функции множества $Q$, называем позитивным замыканием множества $Q$ и обозначаем $\operatorname{Pos}[Q]$. Множества вида $\operatorname{Pos}[Q]$ называем позитивно замкнутыми классами. Известно [4], что всякий позитивно замкнутый класс замкнут относительно операции суперпозиции и содержит все селекторные функции (проекции). Понятия позитивно порождающей системы и позитивно предполного класса аналогичны соответствующим понятиям для операции суперпозиции.

Теорема 1 доказана в сотрудничестве с С. А. Латушкиным.

Теорема 1. Пусть $k \geqslant 2, Q \subseteq P_{k}, u f \in P_{k}$. Тогда $f \in \operatorname{Pos}[Q]$ в том и только том случае, когда $\operatorname{End}(Q) \subseteq \operatorname{End}(f)$.

Доказательство. Необходимость утверждения установлена в теореме 1 из [8]. Поэтому далее будем доказывать достаточность утверждения.

Заметим, что в доказательстве можно ограничиться лишь конечными множествами $Q$. В самом деле, согласно результатам из [6], всякий позитивно замкнутый класс из $P_{k}$ позитивно порождается множеством всех своих функций, зависящих от $k$ переменных. Поэтому, если $Q$ - бесконечное множество функций, то для подходящего конечного подмножества $Q^{\prime} \subset Q$ справедливо соотношение

$$
\operatorname{Pos}\left[Q^{\prime}\right]=\operatorname{Pos}[Q] \text {. }
$$

В силу упомянутой теоремы 1 из [8], множества $\operatorname{End}\left(Q^{\prime}\right)$ и $\operatorname{End}(Q)$ совпадают. Следовательно, вместо $\operatorname{End}(Q)$ в доказательстве можно использовать $\operatorname{End}\left(Q^{\prime}\right)$.

Для любой функции $g \in P_{k}^{(m)}$ обозначим через $\operatorname{Gr}(g)$ график функции $g$, то есть

$$
\operatorname{Gr}(g)=\left\{\left(x_{0}, x_{1}, \ldots, x_{m}\right): x_{0}=g\left(x_{1}, \ldots, x_{m}\right)\right\} .
$$

Пусть множество $Q$ конечно и $\operatorname{End}(Q) \subseteq \operatorname{End}(f)$, где $f \in P_{k}^{(n)}$. Определим позитивную формулу $\Phi$ над $Q$, которая будет выражать отношение $x_{0}=f\left(x_{1}, \ldots, x_{n}\right)$. Формула $\Phi$ имеет вид дизъюнкции $\bigvee_{a \in \operatorname{Gr}(f)} R_{a}$, где вектор $a=\left(a_{0}, a_{1}, \ldots, a_{n}\right)$ пробегает все наборы из графика $\operatorname{Gr}(f)$, а $R_{a}$ представима в форме $\left(\exists z_{0}\right)\left(\exists z_{1}\right) \ldots\left(\exists z_{k-1}\right)\left(C_{a} \& F\right)$. В свою очередь, формулы $C_{a}$ и $F$ имеют вид

$$
\begin{aligned}
C_{a} & =\underset{0 \leqslant i \leqslant n}{\&}\left(z_{a_{i}}=x_{i}\right), \\
F & =\bigotimes_{g \in Q} \underset{\left(b_{1}, \ldots, b_{m}\right) \in E_{k}^{m}}{\mathbb{Z}}\left(g\left(z_{b_{1}}, \ldots, z_{b_{m}}\right)=z_{g\left(b_{1}, \ldots, b_{m}\right)}\right),
\end{aligned}
$$

где функция $g$ зависит от $m$ переменных.

Предположим, что $a \in \operatorname{Gr}(f)$. Рассмотрим значение дизъюнктивного слагаемого $R_{a}$ формулы $\Phi$ на наборе $a$. В качестве значений связанных переменных $z_{0}, z_{1}, \ldots, z_{k-1}$ формулы $R_{a}$ выберем, соответственно, $0,1, \ldots, k-1$. Тогда, очевидно, при любом $i \leqslant n$ будет выполняться равенство $z_{a_{i}}=a_{i}$. Кроме того, равенство

$$
g\left(z_{b_{1}}, \ldots, z_{b_{m}}\right)=z_{g\left(b_{1}, \ldots, b_{m}\right)}
$$

формулы $F$ примет тождественный вид $g\left(b_{1}, \ldots, b_{n}\right)=g\left(b_{1}, \ldots, b_{n}\right)$. Таким образом, формула $R_{a}$ оказывается истинной на наборе $a$. 
Пусть теперь формула $\Phi$ истинна на некотором наборе $\left(x_{0}^{\prime}, x_{1}^{\prime}, \ldots, x_{n}^{\prime}\right)$ значений переменных $x_{0}, x_{1}, \ldots, x_{n}$. Тогда на данном наборе должна быть истинной некоторая формула $R_{a}$. Пусть значениями связанных переменных $z_{0}, z_{1}, \ldots, z_{k-1}$, выполняющими формулу $R_{a}$, будут значения $c_{0}, c_{1}, \ldots, c_{k-1}$. Определим одноместную функцию $h$ равенством

$$
h(i)=c_{i} .
$$

С использованием функции $h$ преобразуем формулы $C_{a}$ и $F$. Формула $C_{a}$ приобретает вид $\&_{0 \leqslant i \leqslant n}\left(h\left(a_{i}\right)=x_{i}^{\prime}\right)$, из которого следует, что набор $\left(x_{0}^{\prime}, x_{1}^{\prime}, \ldots, x_{n}^{\prime}\right)$ совпадает с набором $\left(h\left(a_{0}\right), h\left(a_{1}\right), \ldots, h\left(a_{n}\right)\right)$. Далее, формула $F$ приводится к виду

$$
\&_{g \in Q} \&_{\left(b_{1}, \ldots, b_{m}\right) \in E_{k}^{m}}\left(g\left(h\left(b_{1}\right), \ldots, h\left(b_{m}\right)\right)=h\left(g\left(b_{1}, \ldots, b_{m}\right)\right)\right) .
$$

Очевидно, что полученная формула в точности выражает тот факт, что функция $h$ является эндоморфизмом множества функций $Q$.

Итак, формула $R_{a}$ истинна только на наборе $\left(h\left(a_{0}\right), h\left(a_{1}\right), \ldots, h\left(a_{n}\right)\right)$, и этот набор входит в график функции $f$, поскольку $\left(a_{0}, a_{1}, \ldots, a_{n}\right) \in \operatorname{Gr}(f)$ и $h \in \operatorname{End}(Q) \subseteq \operatorname{End}(f)$. Значит, формула $R_{a}$ “выделяет” лишь наборы из множества $\operatorname{Gr}(f)$, и теорема доказана.

Следствие 1. При любом $k \geqslant 2$ различные позитивно замкнутые классы в $P_{k}$ имеют различные полугруппь эндоморфизмов.

Обозначим через $T_{k}$ множество всех одноместных функций из $P_{k}$, которое рассматривается далее как полугруппа с операцией композиции (суперпозиции) и единицей тождественной функцией. Если $T \subseteq T_{k}$, то через $\operatorname{Gr}(T)$ обозначаем множество графиков всех функций, принадлежащих $T$.

Обозначим через $\Pi_{k}$ множество всех отношений на множестве $E_{k}$. На множестве $\Pi_{k}$ определим операции конъюнкции и проектирования. Пусть $r_{1}$ и $r_{2}$ есть, соответственно, $m$-местное и $n$-местное отношения на $E_{k}$. Конъюнкцией отношений $r_{1}, r_{2}$ назовем $(m+n)$ местное отношение, определяемое формулой $r_{1}\left(x_{1}, \ldots, x_{m}\right) \& r_{2}\left(x_{m+1}, \ldots, x_{m+n}\right)$. Проекцией отношения $r_{1}\left(x_{1}, \ldots, x_{m}\right)$ по переменной $x_{i}, 1 \leqslant i \leqslant m$, назовем $(m-1)$-местное отношение, которое определяется формулой $\left(\exists x_{i}\right) r_{1}\left(x_{1}, \ldots, x_{i-1}, x_{i}, x_{i+1}, \ldots, x_{m}\right)$, где переменная $x_{i}$ под квантором существования пробегает множество $E_{k}$. Элементарной диагональю назовем любое отношение вида $x_{i}=x_{j}$.

Пусть $R \subseteq \Pi_{k}$. Замыканием множества $R$ назовем наименьшее множество отношений из $\Pi_{k}$, которое содержит все элементарные диагонали, все отношения из $R$ и замкнуто относительно операций конъюнкции, проектирования, а также перестановки, переименования и отождествления переменных.

Пусть $r \in \Pi_{k}^{(m)}$ и $f \in P_{k}^{(n)}$. Говорят, что функция $f$ сохраняет отношение $r$, если для любых $n$ наборов $\left(a_{11}, \ldots, a_{m 1}\right), \ldots,\left(a_{1 n}, \ldots, a_{m n}\right)$, удовлетворяющих отношению $r$, набор $\left(f\left(a_{11}, \ldots, a_{1 n}\right), \ldots, f\left(a_{m 1}, \ldots, a_{m n}\right)\right)$ также удовлетворяет отношению $r$. Множество всех функций, сохраняющих отношение $r$, обозначим через $\operatorname{Pol}(r)$. Если $R \subseteq \Pi_{k}$, то положим

$$
\operatorname{Pol}(R)=\bigcap_{r \in R} \operatorname{Pol}(r)
$$

Множество всех отношений, которые сохраняет функция $f$, обозначим через $\operatorname{Inv}(f)$. Если $F \subseteq P_{k}$, то положим

$$
\operatorname{Inv}(R)=\bigcap_{f \in F} \operatorname{Inv}(f)
$$


Известно (см., например, [1]), что для любого множества $R \subseteq \Pi_{k}$ множество $\operatorname{Pol}(R)$ является замкнутым (относительно операции суперпозиции) классом функций, и для любого множества $F \subseteq P_{k}$ множество $\operatorname{Inv}(F)$ является замкнутым классом отношений.

Теорема 2. При любом $k \geqslant 2$ подполугруппа $T$ полугруппь $T_{k}$ имеет вид $\operatorname{End}(Q)$, где $Q$ - некоторый подходящий позитивно замкнутый класс из $P_{k}$, в том и только том случае, когда Т совпадает с множеством всех (одноместных) функиий, графики которых лежат в замыкании множества отночений $\operatorname{Gr}(T)$.

Доказательство. Пусть $Q-$ позитивно замкнутый класс, $Q \subseteq P_{k}$. Известно (и это отмечается в [8]), что для произвольного множества функций $Q$ множество $\operatorname{End}(Q)$ является полугруппой с операцией композиции и единицей - тождественной функцией. Вместе с тем, множество $\operatorname{Pol}(\operatorname{Gr}(\operatorname{End}(Q)))$ состоит в точности из всех функций класса $P_{k}$, которые имеют эндоморфизмы полугруппы $\operatorname{End}(Q)$. Значит, в силу теоремы 1 множество $\operatorname{Pol}(\operatorname{Gr}(\operatorname{End}(Q)))$ есть позитивное замыкание позитивно замкнутого класса $Q$, то есть

$$
\operatorname{Pol}(\operatorname{Gr}(\operatorname{End}(Q)))=Q
$$

Из этого равенства видно, что позитивно замкнутый класс $Q$ определяется, в смысле функтора Pol, конечным множеством двуместных отношений, имеющих вид графиков одноместных функций. Однако множество $\operatorname{Inv}(Q)$ всех отношений, которые сохраняются всеми функциями класса $Q$, содержит диагонали и замкнуто относительно операций конъюнкции и проектирования [1]. Отсюда вытекает, что множество $\operatorname{Gr}(\operatorname{End}(Q))$ содержит графики всех одноместных функций, которые можно получить из диагоналей и графиков функций $\operatorname{End}(Q)$ с помощью применения операций \& и $\exists$.

Обратно, пусть подполугруппа $T$ полугруппы $T_{k}$ удовлетворяет условиям теоремы. Согласно [1], множество $\operatorname{Inv}(\operatorname{Pol}(\operatorname{Gr}(T)))$ получается замыканием множества отношений $\operatorname{Gr}(T)$. Поэтому, в силу условий теоремы, множество $\operatorname{Gr}(T)$ совпадает с множеством всех двуместных отношений из $\operatorname{Inv}(\operatorname{Pol}(\operatorname{Gr}(T)))$, являющихся графиками одноместных функций. Значит, по определению функтора Inv, полугруппа $T$ состоит из всех эндоморфизмов множества $\operatorname{Pol}(\operatorname{Gr}(T))$. В силу теоремы 1 и определения функтора Pol, множество $\operatorname{Pol}(\mathrm{Gr}(T))$ будет являться позитивно замкнутым классом. Это завершает доказательство теоремы.

Для произвольной функции $g \in P_{k}^{(1)}$ обозначим через $\operatorname{PG}(g)$ множество $\operatorname{Pol}(\operatorname{Gr}(g))$. Отметим, что множество $\mathrm{PG}(g)$ замкнуто относительно суперпозиции и состоит из всех функций класса $P_{k}$, имеющих эндоморфизм $g$ (но, возможно, и другие эндоморфизмы).

Пусть $g-$ перестановка на множестве $E_{k}$ и $n \geqslant 1$. Назовем $n$-орбитой функции $g$ наименьшее множество наборов из $E_{k}^{n}$, которое вместе с каждым набором $\left(a_{1}, \ldots, a_{n}\right)$ содержит также набор $\left(g\left(a_{1}\right), \ldots, g\left(a_{n}\right)\right)$.

Функция $g$ из $P_{k}^{1)}$ называется идемпотентной, если

$$
g(g)=g .
$$

Теорема 3. Для любого $k \geqslant 2$ позитивно замкнутый класс $Q \subseteq P_{k}$ является позитивно предполным в $P_{k}$ в том и только том случае, когда $Q$ имеет вид $\mathrm{PG}(g)$, где $g$ - либо идемпотентная функция, отличная от тождественной функиии, либо перестановка на $E_{k}$, которая разлагается в произведение циклов одной и той же простой длинь $u$, возможно, ииклов длины 1 , число которых в этом случае должно быть не менее двух. 
Доказательство. Если $g$ - идемпотентная функция, отличная от тождественной функции, то позитивная предполнота класса $\mathrm{PG}(g)$ установлена в [8]. Если $g$ - перестановка на $E_{k}$, которая разлагается в произведение циклов одной и той же простой длины, то позитивная предполнота класса $\mathrm{PG}(g)$ доказана в [6].

Предположим, что $g-$ перестановка на $E_{k}$ и ее цикловое разложение состоит из циклов одной и той же простой длины и циклов длины 1. Пусть одноэлементные циклы перестановки $g$ суть $a_{1}, \ldots, a_{s}$. Если $s=1$, то, как нетрудно видеть, всякая функция, сохраняющая отношение $y=g(x)$ (график функции $g$ ), сохраняет также константу $a_{1}$ (т.е. график функции-константы $\left.a_{1}\right)$. Таким образом, класс $\operatorname{PG}(g)$ целиком содержится в позитивно предполном классе $\mathrm{PG}\left(a_{1}\right)$, который определяется идемпотентной функциейконстантой $a_{1}$. Следовательно, класс $\mathrm{PG}(g)$ в данном случае не может быть позитивно предполным.

Пусть теперь $s \geqslant 2$. Для простоты будем считать, что цикловое разложение перестановки $g$ имеет вид $(01 \ldots p-1)(p \ldots 2 p-1) \ldots(m p \ldots(m+1) p-1)((m+1) p) \ldots(k-1)$, где $p-$ простое число, $m \geqslant 0$ и $(m+1) p<k-1$. Нетрудно убедиться в том, что классу $\operatorname{PG}(g)$ принадлежат функции-константы $(m+1) p, \ldots, k-1$.

Покажем далее, что для любых $a, b \leqslant(m+1) p-1$ в класс $\mathrm{PG}(g)$ входит такая функция $g_{a b}(x)$, что $g_{a b}(a)=b$. В самом деле, если $a \in\{i p, \ldots,(i+1) p-1\}$ и $b \in\{j p, \ldots,(j+1) p-1\}$, то положим $g_{a b}(a)=b, g_{a b}(a+1)=b+1, \ldots, g_{a b}(a+p-1)=$ $b+p-1$, где сложение в левых частях равенств проводится в цикле $(i p \ldots(i+1) p-1)$, а в правых частях - в цикле $(j p \ldots(j+1) p-1)$, так что, например, $(i+1) p-1+1=i p$. Для значений $x$, не принадлежащих множеству $\{i p, \ldots,(i+1) p-1\}$, полагаем $g_{a b}(x)=x$.

Пусть $f\left(x_{1}, \ldots, x_{n}\right)$ - произвольная функция, не принадлежащая классу PG $(g)$. Покажем, что система функций $\mathrm{PG}(g) \cup\{f\}$ позитивно полна в классе $P_{k}$. Тем самым будет доказана позитивная предполнота класса $\mathrm{PG}(g)$.

Ввиду соотношения $f \notin \mathrm{PG}(g)$, найдется такой набор $\left(b_{1}, \ldots, b_{n}\right) \in E_{k}^{n}$, что

$$
f\left(g\left(b_{1}\right), \ldots, g\left(b_{n}\right)\right) \neq g\left(f\left(b_{1}, \ldots, b_{n}\right)\right) .
$$

Пусть $a \leqslant(m+1) p-1$. Для любого $l, 1 \leqslant l \leqslant n$, обозначим через $g_{a b_{l}}^{\prime}$ функцию $g_{a b_{l}}$, если $b_{l} \leqslant(m+1) p-1$, и функцию-константу $b_{l}$, если $b_{l} \geqslant(m+1) p$. Положим

$$
f_{a}(x)=f\left(g_{a b_{1}}^{\prime}(x), \ldots, g_{a b_{n}}^{\prime}(x)\right) .
$$

Функция $f_{a}$ получена суперпозицией функций множества $\operatorname{PG}(g) \cup\{f\}$ и, кроме того, в силу (1)

$$
f_{a}(g(a)) \neq g\left(f_{a}(a)\right)
$$

так как функции $g_{a b_{1}}^{\prime}, \ldots, g_{a b_{n}}^{\prime}$ принадлежат классу PG $(g)$ и потому при любом $l$

$$
g_{a b_{l}}^{\prime}(g(a))=g\left(g_{a b_{l}}^{\prime}(a)\right) .
$$

Таким образом, при любом $a \leqslant(m+1) p-1$ нами получена функция $f_{a}$, для которой неравенство (2) опровергает ее принадлежность классу $\mathrm{PG}(g)$ на примере точек $a$ и $g(a)$.

Мы хотим далее определить аналогичные функции, но для большего расстояния между точками “опровержения” $а$ и $g(g(a)), a$ и $g(g(g(a)))$ и т. д. С этой целью при $p>2$ зафиксируем $q, 2 \leqslant q<p$, и рассмотрим, например, для $a \in\{0,1, \ldots, p-1\}$ значения функции $f_{a}$ в точках $a, a+q, a+2 q, \ldots, a+l q$, где арифметические действия выполняются по модулю $p$ и

$$
l q \equiv 1 \quad(\bmod p)
$$


Поскольку

$$
g(x)=x+1 \quad(\bmod p)
$$

на множестве $\{0,1, \ldots, p-1\}$, а согласно (2)

$$
f_{a}(a+l q) \neq f_{a}(a)+1,
$$

найдется пара “соседних” точек $a+i q$ и $a+(i+1) q$, в которых будет нарушаться условие принадлежности функции $f_{a}$ классу $\mathrm{PG}(g)$ :

$$
f_{a}(a+(i+1) q) \neq f_{a}(a+i q)+q .
$$

Таким образом, если мы хотим для данного значения $a$ определить функцию, у которой в точках $a$ и $a+q$ достигается опровержение ее принадлежности классу $\operatorname{PG}(g)$, то можно, например, взять функцию $f_{a}(x+i q)$.

Для любых $a \leqslant(m+1) p-1$ и $q, 1 \leqslant q<p$, обозначим через $f_{a q}(x)$ функцию, построенную на основе функции $f_{a}$ описанным выше способом для точек $a$ и $g^{q}(a)$, где $g^{q}-q$-кратная суперпозиция функции $g$. Положим $r=(m+1) p(p-1)$. Рассмотрим набор из $r+1$ функций

$$
\begin{array}{r}
\left(x, f_{01}(x), \ldots, f_{p-1, p-1}(x), f_{p 1}(x), \ldots, f_{2 p-1, p-1}(x), \ldots,\right. \\
\left.\quad f_{m p, 1}(x), \ldots, f_{(m+1) p-1, p-1}(x)\right) .
\end{array}
$$

Заметим, что набор функций (3) при $x=0,1, \ldots, k-1$ дает $k$ наборов из $E_{k}^{r+1}$, которые принадлежат различным $(r+1)$-орбитам функции $g$. В самом деле, если $a, b \in\{i p, i p+1, \ldots,(i+1) p-1\}$ и $a \neq b$, то элемент $a$ переводится в элемент $b$ подходящей $q$-й степенью перестановки $g$. Вместе с тем, по построению, соответствующая функция $f_{a q}$ набора (3) обеспечит невхождение в одну и ту же 2-орбиту функции $g$ наборов $\left(a, f_{a q}(a)\right),\left(b, f_{a q}(b)\right)$. Если элементы $a, b$ входят в различные циклы циклового разложения перестановки $g$, то равенство $g^{q}(a)=b$, очевидно, невозможно ни при каком $q$ (используем принадлежность переменной $x$ последовательности (3)).

То же самое получаем в случае, когда один из элементов $a, b$ не превосходит величины $(m+1) p-1$, а другой больше этой величины, либо когда оба элемента больше, чем $(m+1) p-1$.

Установленное свойство набора (3) позволяет определить в классе $\mathrm{PG}(g)$ такую функцию $h$ от $r+1$ переменных, что при любом $x$ выполняется равенство

$$
h\left(x, f_{01}(x), \ldots, f_{(m+1) p-1, p-1}(x)\right)=0 .
$$

Итак, функция, стоящая в левой части этого равенства, тождественно равна 0. С помощью функций $g_{0 b}$ получаем остальные константы $1,2, \ldots,(m+1) p-1$. Поскольку константы $(m+1) p, \ldots, k-1$ присутствуют в классе $\mathrm{PG}(g)$, а система всех констант позитивно полна в классе $P_{k}[6]$, заключаем, что система функций $\operatorname{PG}(g) \cup\{f\}$ также позитивно полна в классе $P_{k}$.

Перейдем к доказательству второй части теоремы. Пусть $Q-$ произвольный позитивно предполный в $P_{k}$ класс. Поскольку, очевидно,

$$
\operatorname{End}\left(P_{k}\right)=\{x\},
$$

согласно уже доказанной теореме 1 получаем, что

$$
\operatorname{End}(Q) \neq\{x\}
$$


Предположим, что в $\operatorname{End}(Q)$ входит неинъективная функция $g^{\prime}$. Тогда с помощью суперпозиции из нее можно получить идемпотентную функцию $g$, отличную от тождественной функции. Очевидно, что $g \in \operatorname{End}(Q)$. Вместе с тем, позитивно замкнутый класс $\operatorname{PG}(g)$ включает в себя, разумеется, весь класс $Q$. Как установлено выше, класс $\operatorname{PG}(g)$ позитивно предполон в $P_{k}$. Значит, в этом случае класс $Q$ совпадает с классом $\mathrm{PG}(g)$.

Предположим, далее, что все функции из $\operatorname{End}(Q)$ суть перестановки на $E_{k}$. Поскольку в данном случае $\operatorname{End}(Q)$ образует группу, в ней найдется такая перестановка $g$, которая разлагается в произведение циклов одной и той же простой длины и, возможно, циклов длины 1. Из соотношения $g \in \operatorname{End}(Q)$ следует, что $\operatorname{PG}(g) \supseteq Q$. Далее воспользуемся первой частью доказываемой теоремы. Если в цикловом разложении перестановки $g$ отсутствуют циклы длины 1 либо их число не менее двух, то $\mathrm{PG}(g)$ - позитивно предполный в $P_{k}$ класс и, следовательно,

$$
\operatorname{PG}(g)=Q \text {. }
$$

В противном случае класс $\mathrm{PG}(g)$ целиком содержится в позитивно предполном классе вида $\mathrm{PG}(a)$, где $a-$ функция-константа. Однако позитивно предполный класс $Q$ не может строго содержаться в позитивно предполном классе $\mathrm{PG}(a)$. Противоречие завершает доказательство теоремы.

Отметим, что все три позитивно предполных класса в $P_{2}$ найдены ранее в [4], а все десять позитивно предполных классов в $P_{3}$ найдены в [6].

\section{Список литературы}

1. Боднарчук В. Г., Калужнин Л. А., Котов В. Н., Ромов Б. А., Теория Галуа для алгебр Поста. Кибернетика (1969), № 3, 5, 1-10, 1-9.

2. Данильченко А. Ф., О параметрической выразимости функций трехзначной логики. Алгебра $u$ логика (1977) 16, №4, 397-416.

3. Кузнецов А. В., О средствах для обнаружения невыводимости и невыразимости. В сб.: Логический вывод. Наука, Москва, 1979, с. 5-33.

4. Марченков С. С., О выразимости функций многозначной логики в некоторых логико-функциональных языках. Дискретная математика (1999) 11, №4, 110-126.

5. Марченков С. С., Замкнутые классы булевых функиий. Физматлит, Москва, 2000.

6. Марченков С. С., Критерий позитивной полноты в трехзначной логике. Дискретный анализ $и$ исследование операчий, Сер. 1 (2006) 13, №3, 27-39.

7. Марченков С. С., Дискриминаторные позитивно замкнутые классы трехзначной логики. Дискретный анализ и исследование операчий, Сер. 1 (2007) 14, №3, 53-66.

8. Марченков С. С., О замкнутых классах функций $k$-значной логики, определяемых одним эндоморфизмом. Дискретный анализ и исследование операций (2009) 16, №6, 52-67.

9. Марченков С. С., Позитивно замкнутые классы трехзначной логики, порождаемые одноместными функциями. Дискретная математика (2009) 21, №3, 37-44.

10. Янов Ю. И., Мучник А. А., О существовании $k$-значных замкнутых классов, не имеющих базиса. Доклады АН СССР (1959) 127, №1, 44-46.

11. Barris S., Willard R., Finitely many primitive positive clones. Proc. Amer. Math. Soc. (1987) 101, 427-430. 